\title{
Second Order Scheme for the Spatially Homogeneous Boltzmann Equation with Maxwellian Molecules
}

\author{
Jens Struckmeier and Konrad Steiner \\ Department of Mathematics \\ University of Kaiserslautern \\ P.O. Box 3049 \\ 67653 Kaiserslautern \\ Germany
}

\begin{abstract}
In the standard approach, particle methods for the Boltzmann equation are obtained using an explicit time discretization of the spatially homogeneous Boltzmann equation. This kind of discretization leads to a restriction on the discretization parameter as well as on the differential cross section in the case of the general Boltzmann equation. Recently, it was shown, how to construct an implicit particle scheme for the Boltzmann equation with Maxwellian molecules.

The present paper combines both approaches using a linear combination of explicit and implicit discretizations. It is shown, that the new method leads to a second order particle method, when using an equiweighting of explicit and implicit discretization.
\end{abstract}




\section{Introduction}

Particle methods are the most efficient numerical tools to describe rarefied gas flows (see [Bird, 1994] and chapter 10 in [Cercignani et al., 1994]) and they may be mainly divided into two quite different branches.

The first branch [B, 1994] considers rarefied gas flows from the point of real applications: real-gas effects, like internal energies and chemically reacting flows, play the more important role than numerical aspects. The particle method (DSMC) is based on a stochastic simulation of $N$-particle systems.

Particle methods concerning the second branch [Babovsky, 1989], [Neunzert et al., 1991], [Neunzert \& Struckmeier, 1994] (Low-Discrepancy Methods or Finite Pointset Methods) are derived from the Boltzmann equation by classical discretization techniques. The main feature is that one is able to approximate (or simulate) the collision integral of the Boltzmann equation (see also [Bobylev \& Struckmeier, 1994]). The discretization used is an explicit one and this restricts the magnitude of the time step. To overcome this difficulty, which is typical for kinetic equation, attempts to use an semi-implicit discretization were given in [Russo \& Caflisch, 1994].

Recently it was shown [B \& S, 1994] how to work with a fully implicit discretization for the Boltzmann equation with Maxwellian molecules. For this approach the time steps may be arbitrary large. In [Struckmeier, 1995] the author developed a particle method for the fully implicit discretization.

Using implicit particle schemes the timestep may be enlarged by an order of magnitude and this is most relevant for computations at low Knudsen numbers. Nevertheless, the implicit scheme is still only of first order in time.

Besides the fact, that for most applications in rarefied gas dynamics, one is mainly interested in the stationary state, it is important to improve the accuracy of particle schemes from first to second order in time.

In the following we present a mixed explicit-implicit discretization technique for the Boltzmann equation with Maxwellian molecules, which leads to a second order scheme.

In section 2 we shortly recall the explicit and implicit discretization techniques for the spatially homogeneous Boltzmann equation. The next section presents a second order scheme obtained by combining the explicit and implicit discretization. Moreover, we show, how to realize the new scheme by a particle method. Some numerical results are presented in section 4 . 


\section{Time Discretization Techniques}

The starting point for our considerations is the (spatial uniform) initial value problem

$$
f_{t}=I f, \quad f(0, v)=\varphi(v),\|\varphi\|_{1}=1
$$

where $\|\cdot\|_{1}$ denotes the $\mathcal{L}_{1}-$ norm on $I R^{3}$,

$$
I f=\int_{\mathbb{R}^{3}} \int_{S_{+}^{2}}\left\{f\left(v^{\prime}\right) f\left(w^{\prime}\right)-f(v) f(w)\right\} \mathrm{d} \omega(\eta) \mathrm{d} w
$$

and

$$
\begin{aligned}
S_{+}^{2} & =\left\{\eta \in I R^{3}:|\eta|=1,\langle v-w, \eta\rangle>0\right\} \\
v^{\prime} & =v-\eta\langle v-w, \eta\rangle \\
w^{\prime} & =w+\eta\langle v-w, \eta\rangle
\end{aligned}
$$

Equation (1) describes a space uniform rarefied gas flow consisting of (pseudo) Maxwellian molecules. In this special model, the collision probability is independent of the relative velocity and the collision mechanism depends on some probability measure $\omega(\eta)$ on $S_{+}^{2}$.

Because of

$$
\int_{\boldsymbol{R}^{3}} I f \mathrm{~d} v=0
$$

we have total mass conservation, i.e.

$$
\|f(t, \cdot)\|_{1}=\|\varphi\|_{1}=1 \quad \forall t \in R_{+}
$$

and equation (1) may be written as

$$
f_{t}=I^{+} f-f, \quad f(0, v)=\varphi(v)
$$

with

$$
I^{+} f=\int_{\boldsymbol{R}^{3}} \int_{S_{+}^{2}} f\left(v^{\prime}\right) f\left(w^{\prime}\right) \mathrm{d} \omega(\eta) \mathrm{d} w
$$

The classical approach to derive particle schemes for the Boltzmann equation is to use an explicit time discretization (here for the first discrete timestep)

$$
\frac{f_{\Delta}-\varphi}{\Delta}=I^{+} \varphi-\varphi
$$


which leads - applying the weak formulation - to

$$
\int_{\mathbb{R}^{3}} \Phi f_{\Delta} \mathrm{d} v=\iint_{\boldsymbol{R}^{3}} \int_{\mathbb{R}^{3}} \int_{S_{+}^{2}}(\Phi \circ T)(v, w, \eta, s) \varphi(v) \varphi(w) \mathrm{d} s \mathrm{~d} \omega(\eta) \mathrm{d} w \mathrm{~d} v
$$

where

$$
T(v, w, \eta, s)=\left\{\begin{array}{lll}
v^{\prime} & : & s \leq \Delta \\
v & : & s>\Delta
\end{array}\right.
$$

and (2) should hold for all functions $\Phi \in \mathcal{C}_{b}\left(I^{3}\right)$, the set of all real-valued, bounded and continuous functions on $I R^{3}$. Concerning more details on this approach we refer the reader to [C et al., 1994] and [N et al., 1994].

This explicit approach is restricted by

$$
\Delta \leq 1
$$

otherwise the positivity of $f_{\Delta}$ is not guaranteed.

\section{Remark 1}

When considering the full Boltzmann equation with an arbitrary scattering kernel $k(\eta,|v-w|)$, i.e. the collision integral is defined as

$$
I f=\iint_{\mathbb{R}^{3}} \int_{S_{+}^{2}} k(\eta,|v-w|)\left\{f\left(v^{\prime}\right) f\left(w^{\prime}\right)-f(v) f(w)\right\} \mathrm{d} \omega(\eta) \mathrm{d} w,
$$

it is moreover necessary to bound the scattering kernel: for a hard sphere gas the scattering kernel is

$$
k(\eta,|v-w|)=|\langle v-w, \eta\rangle|
$$

and this kernel has to be truncated such that

$$
\int_{S_{+}^{2}} k(\eta,|v-w|) \mathrm{d} \omega(\eta) \leq K
$$

for some $K>0$. As a consequence, the restriction on $\Delta$ is more severe then in the case of Maxwellian molecules, especially for flows near to the equilibrium. 
Under some assumptions on the solution $f(t, \cdot)$ of the initial value problem, one may prove that the explicit discretization is a first order scheme.

The main advantage is, that it is quite simple to derive a particle scheme, which converges weak* to the discretized version (1):

The functions $\varphi$ and $f_{\Delta}$ are positive with $\|\varphi\|_{1}=\left\|f_{\Delta}\right\|_{1}=1$. Hence, they may be interpreted as densities of probability measures, which may be approximated by discrete measures (respectively point sets).

Suppose that we approximate the initial condition $\varphi$ by $N$ particles with velocities $v_{1}, \ldots, v_{N}$. Then one has to choose $\frac{N}{2}$ collision pairs, the same number of impact parameters $\eta_{1}, \ldots, \eta_{N / 2}$ and uniformly distributed random numbers $s_{1}, \ldots, s_{N / 2}$ on $[0,1]$. The new velocities at time $t=\Delta$ are then given by the transformation $T$ : if $s_{i}$ is less then $\Delta$ we perform a collision otherwise not. One can show the weak* convergence of the algorithm to the solution of the discretized equation (see for example [C et al.,1994] and the references given in $[\mathrm{N} \& \mathrm{~S}, 1994]$.

The fully implicit discretization

$$
f_{\Delta}=\Delta\left(I^{+} f_{\Delta}-f_{\Delta}\right)+\varphi
$$

may be applied without any restrictions on $\Delta$. Using the iteration

$$
\begin{aligned}
f_{\Delta}^{(n+1)} & =\frac{\Delta}{1+\Delta} I^{+} f_{\Delta}^{(n)}+\frac{1}{1+\Delta} \varphi \\
f_{\Delta}^{(0)} & =\varphi,
\end{aligned}
$$

it can be shown [B \& S, 1994], that the sequence $\left\{f_{\Delta}^{(n)}\right\}_{n \in N}$ converges at least for the moments of the distribution function to the unique fixpoint of (3).

In analogy to the classical discretization techniques for ordinary differential equations one may expect to improve the accuracy to second order by taking a linear combination of explicit and implicit discretization. In the following we will investigate such an approach and show how to construct a second order particle scheme.

\section{Second Order Particle Schemes}

Suppose $\alpha \in[0,1]$, then we consider the mixed discretization for the initial value problem (1) (again for the first discrete timestep)

$$
\frac{f_{\Delta}-\varphi}{\Delta}=(1-\alpha) I \varphi+\alpha I f_{\Delta}
$$


If $\alpha=1$ the discretization is fully implicit, if $\alpha=0$ fully explixit.

Under the following assumption on the solution $f(t, \cdot)$ of the initial value problem, one can prove that the mixed discretization, taking $\alpha=\frac{1}{2}$, is a second order scheme in time:

(B) The solution $f(t, \cdot)$ is two times differentiable with respect to $t$ on $I R$ and for $t \in I R, \Delta>0$ their exist two $\mathcal{L}_{1}$-bounded functions

$$
\mathcal{A}_{\Delta, t}: I^{3} \rightarrow I R
$$

and

$$
\mathcal{B}_{\Delta, t}: I^{3} \rightarrow I R
$$

with $\left\|\mathcal{A}_{\Delta, t}\right\|_{1} \leq M_{1}<\infty$ and $\left\|\mathcal{B}_{\Delta, t}\right\|_{1} \leq M_{2}<\infty$, such that for all $v \in \mathbb{I R}^{3}$

$$
\begin{aligned}
f(t+\Delta, v) & =f(t, v)+\Delta f_{t}(t, v)+\frac{\Delta^{2}}{2} f_{t t}(t, v)+\Delta^{3} \mathcal{A}_{\Delta, t}(v) \\
f_{t}(t+\Delta, v) & =f_{t}(t, v)+\Delta f_{t t}(t, v)+\Delta^{2} \mathcal{B}_{\Delta, t}(v)
\end{aligned}
$$

\section{Theorem 1}

Assume that the solution $f(t, \cdot)$ of the initial value problem (1) fulfills (B). Consider the mixed discretization

$$
f_{\Delta, t}(v)=f(t, v)+(1-\alpha)(I f)(t, v)+\alpha\left(I f_{\Delta, t}\right)(v)
$$

then, if $\alpha=\frac{1}{2}$,

$$
\left\|f_{\Delta, t}(\cdot)-f(t+\Delta, \cdot)\right\|_{1}=O\left(\Delta^{3}\right)
$$

i.e. the mixed discretization with $\alpha=\frac{1}{2}$ is second order in time.

\section{Proof}

Suppose $\alpha=\frac{1}{2}$. If we are able to show that there exists $c_{1}, c_{2}>0$ such that

$$
\left\|f_{\Delta, t}(\cdot)-f(t+\Delta, \cdot)\right\|_{1} \leq c_{1} \Delta\left\|f_{\Delta, t}(\cdot)-f(t+\Delta, \cdot)\right\|_{1}+c_{2} \Delta^{3},
$$

we have (assuming $\left\|f_{\Delta, t}(\cdot)-f(t+\Delta, \cdot)\right\|_{1}>0$ )

$$
1 \leq c_{1} \Delta+c_{2} \frac{\Delta^{3}}{\left\|f_{\Delta, t}(\cdot)-f(t+\Delta, \cdot)\right\|_{1}}
$$

from which we conclude that

$$
\left\|f_{\Delta, t}(\cdot)-f(t+\Delta, \cdot)\right\|_{1}=O\left(\Delta^{3}\right)
$$


which completes the proof.

Denote

$$
e_{\Delta, t}=\left\|f_{\Delta, t}(\cdot)-f(t+\Delta, \cdot)\right\|_{1}
$$

By assumption $f(t, \cdot)$ is a solution of (1) and we estimate using (5)

$$
e_{\Delta, t} \leq \frac{\Delta}{2}\left\|\left(I f_{\Delta, t}\right)(\cdot)-(I f)(t, \cdot)-\Delta f_{t t}(t, \cdot)\right\|_{1}+\Delta^{3}\left\|\mathcal{A}_{\Delta, t}(\cdot)\right\|_{1}
$$

Now using (6) we have

$$
\begin{aligned}
& \left\|\left(I f_{\Delta, t}\right)(\cdot)-(I f)(t, \cdot)-\Delta f_{t t}(t, v)\right\|_{1} \leq \\
& \quad\left\|\left(I f_{\Delta, t}\right)(\cdot)-(I f)(t+\Delta, \cdot)\right\|_{1}+\Delta^{2}\left\|\mathcal{B}_{\Delta, t}(\cdot)\right\|_{1}
\end{aligned}
$$

and (8) yields

$$
e_{\Delta, t} \leq \frac{\Delta}{2}\left\|\left(I f_{\Delta, t}\right)(\cdot)-(I f)(t+\Delta, \cdot)\right\|_{1}+\Delta^{3}\left(\left\|\mathcal{A}_{\Delta, t}(\cdot)\right\|_{1}+\frac{1}{2}\left\|\mathcal{B}_{\Delta, t}(\cdot)\right\|_{1}\right)
$$

It remains to estimate the first term on the left hand side of (9) using the special structure of the collision integral $I$ :

$$
\begin{aligned}
& \left\|\left(I f_{\Delta, t}\right)(\cdot)-(I f)(t+\Delta, \cdot)\right\|_{1} \leq \\
& \quad\left\|f_{\Delta, t}(\cdot)-f(t+\Delta, \cdot)\right\|_{1}+\left\|\left(I^{+} f_{\Delta, t}\right)(\cdot)-\left(I^{+} f\right)(t+\Delta, \cdot)\right\|_{1}
\end{aligned}
$$

and by straightforward computations

$$
\left\|\left(I^{+} f_{\Delta, t}\right)(\cdot)-\left(I^{+} f\right)(t+\Delta, \cdot)\right\|_{1} \leq 2\left\|f_{\Delta, t}(\cdot)-f(t+\Delta, \cdot)\right\|_{1},
$$

so we have

$$
e_{\Delta, t} \leq 3 \Delta e_{\Delta, t}+\left(\left\|\mathcal{A}_{\Delta, t}(\cdot)\right\|_{1}+\frac{1}{2}\left\|\mathcal{B}_{\Delta, t}(\cdot)\right\|_{1}\right) \Delta^{3},
$$

which fits into the required estimate (7).

It remains to show how the mixed formulation (4) may be solved and we directly follow the iteration technique for the full implicit scheme as described at the end of section 2. Using $I f=I^{+} f-f$ yields

$$
f_{\Delta}=\frac{\alpha \Delta}{1+\alpha \Delta} I^{+} f_{\Delta}+\frac{1}{1+\alpha \Delta} \mathcal{R} \varphi
$$

with

$$
\mathcal{R} \varphi=(1-\alpha) \Delta I^{+} \varphi+(1-(1-\alpha) \Delta) \varphi
$$


To solve (11) we take the iteration

$$
f_{\Delta}^{(n+1)}=\frac{\alpha \Delta}{1+\alpha \Delta} I^{+} f_{\Delta}^{(n)}+\frac{1}{1+\alpha \Delta} \mathcal{R} \varphi
$$

and

$$
f_{\Delta}^{(0)}=\mathcal{R} \varphi
$$

Now, the function $\mathcal{R} \varphi$ is nothing else then an explicit time discretization with starting point $\varphi$ and discrete timestep $(1-\alpha) \Delta$. Hence, to ensure positivity of $\mathcal{R} \varphi$ we have the restriction

$$
\Delta \leq \frac{1}{1-\alpha}
$$

Concerning the convergence of the iteration we have

Lemma 1

$$
\left\|f_{\Delta}^{(n+1)}-f_{\Delta}^{(n)}\right\|_{1} \leq \frac{2 \alpha \Delta}{1+\alpha \Delta}\left\|f_{\Delta}^{(n)}-f_{\Delta}^{(n-1)}\right\|_{1}
$$

\section{Proof}

We estimate using (10)

$$
\begin{aligned}
\left\|f_{\Delta}^{(n+1)}-f_{\Delta}^{(n)}\right\|_{1} & =\frac{\alpha \Delta}{1+\alpha \Delta}\left\|I^{+} f^{(n)}-I^{+} f^{(n-1)}\right\|_{1} \\
& \leq \frac{\alpha \Delta}{1+\alpha \Delta} 2\left\|f^{(n)}-f^{(n-1)}\right\|_{1}
\end{aligned}
$$

Hence by Banach's Fixpoint Theorem we obtain

\section{Theorem 2}

If $\Delta<\frac{1}{\alpha}$, the sequence $\left\{f_{\Delta}^{(n)}\right\}_{n \in N}$ converges in $\mathcal{L}_{1}\left(I^{3}\right)$ to the unique fixpoint of (12).

\section{Remark 2}

Especially, for $\alpha=\frac{1}{2}$, we have a convergence if $\Delta<2$, which fits to the restriction $\Delta \leq 2$ given by (13).

By Lemma 1 we may also estimate the speed of convergence:

\section{Corollary 1}

$$
\left\|f_{\Delta}^{(n+1)}-f_{\Delta}^{(n)}\right\|_{1} \leq \frac{1}{\alpha}\left(\frac{2 \alpha \Delta}{1+\alpha \Delta}\right)^{n+1}
$$




\section{Proof}

Using $\|\mathcal{R} \varphi\|_{1}=1$ we estimate

$$
\left\|f_{\Delta}^{(1)}-f_{\Delta}^{(0)}\right\| \leq \frac{2 \Delta}{1+\alpha \Delta}
$$

and (14) from Lemma 1 by induction.

The second order discretization scheme may be used for quite different numerical methods, like finite difference, finite element scheme or finite pointset schemes.

In the following we describe, how to use the implicit formulation (11) with $\alpha=\frac{1}{2}$ for the construction of a second order particle scheme.

The second order discretization scheme is given by (11) with $\alpha=\frac{1}{2}$, i.e.

$$
f_{\Delta}=\frac{\Delta}{2+\Delta} I^{+} f_{\Delta}+\frac{2}{2+\Delta} \mathcal{R} \varphi
$$

To solve this implicit equation we use the iteration defined in (12) with starting point $\mathcal{R} \varphi$

$$
f_{\Delta}^{(n+1)}=\frac{\Delta}{2+\Delta} I^{+} f_{\Delta}^{(n)}+\frac{2}{2+\Delta} \mathcal{R} \varphi, \quad f^{(0)}=\mathcal{R} \varphi
$$

As already mentioned, $\mathcal{R} \varphi$ is an explicit discretization with time step $\frac{\Delta}{2}$ and initial condition $\varphi$. Hence we may apply the algorithm which was shortly discussed in section 2. For the iteration (15) we may apply the method as defined in [S, 1995].

The complete algorithm may be summarized as follows:

1) Suppose the $N$ particles with velocities $v_{1}, \ldots, v_{N}$ approximate the initial condition $\varphi$ at time $t=0$. Choose a timestep $\Delta<2$.

2) Perform an explicit timestep with parameter $\frac{\Delta}{2}$ to generate an approximation of $\mathcal{R} \varphi$ :

Choose $\frac{N}{2}$ collision pairs out of the set $\left\{v_{1}, \ldots, v_{N}\right\}$ of given velocities. Generate $\frac{N}{2}$ random numbers on $[0,1]$ and the same number of unit vectors $\eta_{i}$, distributed due to the measure $\omega(\eta)$ on the halfsphere $S_{+}^{2}$. For every pair with $s_{i}<\frac{\Delta}{2}$ perform a collision with unit vector $\eta_{i}$. Denote the new set of velocities by $\left\{v_{1}^{*}, \ldots, v_{N}^{*}\right\}$. 
3) Generate the the pointset $\left\{v_{1}^{(0)}, \ldots, v_{N}^{(0)}\right\}$ by

$$
v_{i}^{(0)}=v_{i}^{*}, \quad i=1, \ldots, N
$$

and perform the following iteration process from $(n) \rightarrow(n+1)$ :

Define

$$
L=\frac{2 N}{2+\Delta}
$$

and assume $L \in I N$.

Choose randomly $L$ velocities $\left\{w_{1}^{*}, \ldots, w_{L}^{*}\right\}$ out of the set $\left\{v_{1}^{*}, \ldots, v_{N}^{*}\right\}$ and set

$$
v_{i}^{(n+1)}=w_{i}^{*}, \quad i=1, \ldots, L .
$$

Choose randomly $\frac{N-L}{2}$ collision pairs out of the set $\left\{v_{1}^{(n)}, \ldots, v_{N}^{(n)}\right\}$ and perform a collision with a unit vector distributed due to the measure $\omega(\eta)$ on the halfsphere $S_{+}^{2}$. The resulting velocities complete the set $\left\{v_{1}^{(n+1)}, \ldots, v_{N}^{(n+1)}\right\}$ at the $(n+1)^{\text {th }}$ iteration.

\section{Remark 3}

1) The algorithm given above obviously ensures total mass conservation. On the other hand, momentum and energy are conserved in average. We refer the reader to [S, 1995].

2) The convergence of the algorithm may be investigated using the techniques described in [S, 1995]: applying the central limit theorem and the Borel-Cantelli lemma it is proved that the algorithm converges, almost surely with respect to the required random variates, weak* to the solution of the discretized equation if $N \rightarrow \infty$.

\section{Numerical Example}

We consider the initial value problem

$$
f_{t}=I^{+} f-f
$$

with isotropic scattering, which means that $\omega(\eta)$ is the uniform measure on $S_{+}^{2}$. As initial condition we take the non-isotropic distribution

$$
\varphi(v)=\delta\left(v_{z}\right) \cdot \delta\left(\left|v_{x} v_{x}+v_{y} v_{y}\right|-1\right)
$$


i.e. at time $t=0$ the velocities are uniformly distributed on a circle with radius 1 in the $x y$-plane.

The stationary solution of (16) is the Maxwellian $f_{\infty}(v)=f(t \rightarrow \infty, v)$ with

$$
f_{\infty}(v)=\frac{1}{(2 \pi)^{3 / 2}} \mathrm{e}^{-\frac{|v|^{2}}{2}}
$$

In the following numerical results we use 10.000 particles to approximate the solution of (16) and 50 independent samples to improve the accuracy. For the first order explicit scheme we vary the time discretization between $\Delta=0.05$ up to $\Delta=1.0$, for the second order scheme between $\Delta=0.2$ and $\Delta=2.0$, where the second order scheme is obtained using 5 local iterations per timestep.

Figure 1 illustrates the approximate solution on the time intervall $[0: 10]$ for the second moment in $z$-direction - the integrated quantity

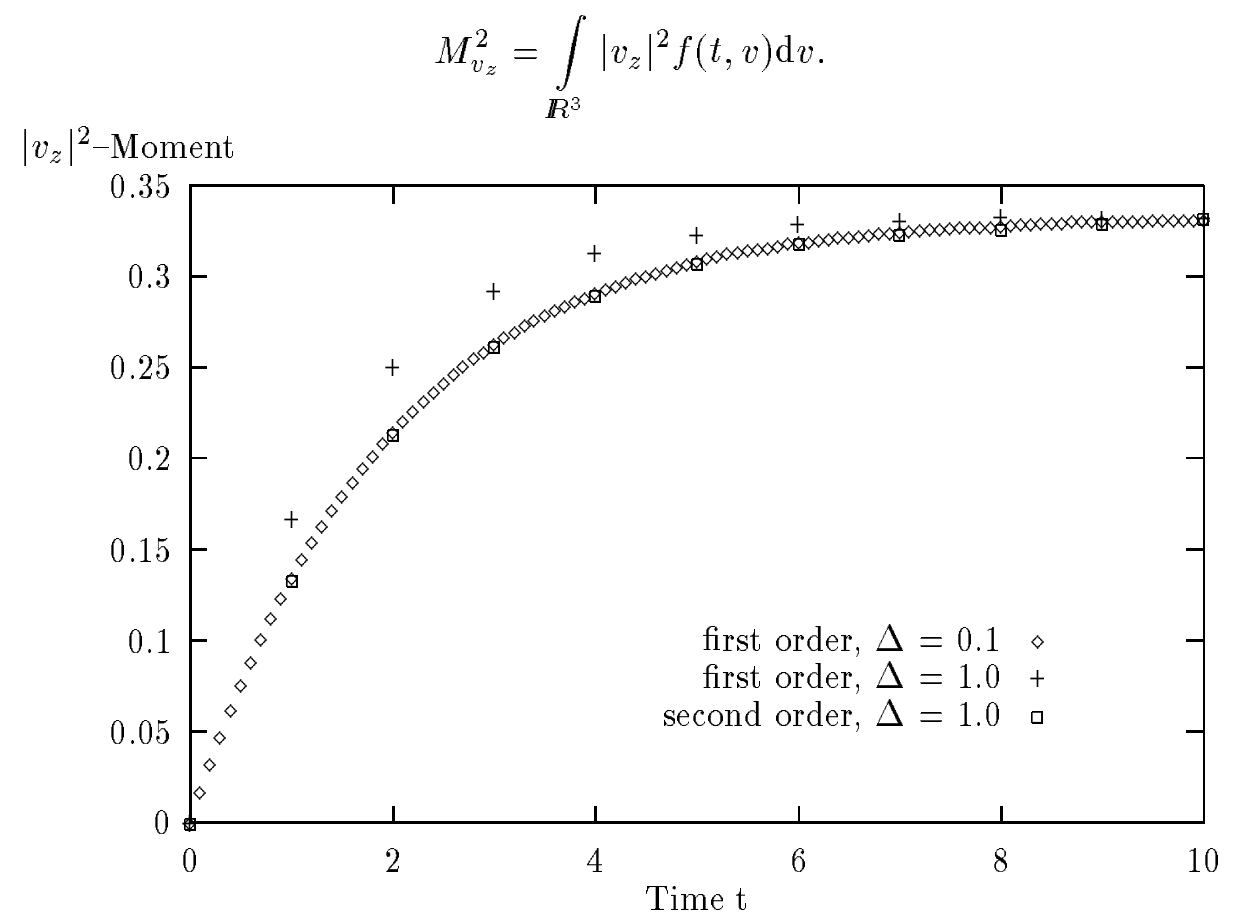

Fig. 1. Instationary solution with first and second order schemes

The agreement between the first order solution with $\Delta=0.1$ and the second order solution with $\Delta=1.0$ is excellent. On the other hand, the first 
order solution with $\Delta=1.0$ shows a strong derivation in the instationary behaviour.

A more detailed investigation is shown in table 1 . Here we compare the numerical value of $M_{v_{z}}^{2}$ at time $t=2$ for the two different schemes and various discretization parameter $\Delta$.

Tab. 1. $\left|v_{z}\right|^{2}-$ Moment at time $t=2$

\begin{tabular}{c|cccccc}
\hline \hline Scheme & $\Delta=0.05$ & $\Delta=0.1$ & $\Delta=0.2$ & $\Delta=0.5$ & $\Delta=1.0$ & $\Delta=2.0$ \\
\hline 1. Order & .212 & .215 & .217 & .228 & .249 & \\
2. Order & & & .211 & .211 & .213 & .222 \\
\hline
\end{tabular}

\section{Remark 4}

In Corollary 1 it was shown that the iteration converges if $\Delta<2$. Nevertheless, the numerical experiments show, that we have a fast convergence if $\Delta=2($ see also table 3$)$.

To clarify, the second order particle scheme is more time-consuming then the corresponding first order explicit - because of the local iterations per timestep (see table 2).

Tab. 2. CPU-times in min:sec

\begin{tabular}{l|cccccc}
\hline \hline Scheme & $\Delta=0.05$ & $\Delta=0.1$ & $\Delta=0.2$ & $\Delta=0.5$ & $\Delta=1.0$ & $\Delta=2.0$ \\
\hline 1. Order & $17: 13$ & $9: 04$ & $5: 00$ & $2: 33$ & $1: 42$ & \\
2. Order & & & $14: 16$ & $6: 53$ & $4: 12$ & $2: 39$ \\
\hline
\end{tabular}

On the other hand, the computational effort of the second order scheme may be reduced by decreasing the number of local iterations per timesteps. Especially, if the parameter $\Delta$ is small the convergence of the local iteration is very fast (compare Corollary 1) and the number of iterations as well as the computational effort may be reduced. 
Tab. 3. $\left|v_{z}\right|^{2}$-Moment at time $t=2$

\begin{tabular}{c|cccc}
\hline \hline Nr. of Iter. & $\Delta=0.2$ & $\Delta=0.5$ & $\Delta=1.0$ & $\Delta=2.0$ \\
\hline 0 & .134 & .139 & .147 & .167 \\
1 & .208 & .205 & .203 & .207 \\
2 & .211 & .211 & .212 & .218 \\
3 & .211 & .211 & .213 & .221 \\
4 & .211 & .211 & .213 & .222 \\
5 & .211 & .211 & .213 & .222 \\
6 & .211 & .211 & .213 & .222 \\
\hline
\end{tabular}

The dependence of $M_{\left|v_{z}\right|}^{2}$ on the local iteration is shown in table 3. Obviously, nearly the same results are obtained using 3 instead of 5 local iterations: the convergence is fast enough over the whole range of discretization parameters between 0.2 and 2.0 .

\section{Remark 5}

The value obtained using zero local iterations is exactly the value using an explicit discretization with time step $\frac{\Delta}{2}$.

The reduction in CPU-time by decreasing the number of local iterations is shown in table 4 .

Tab. 4. CPU-times in min:sec

\begin{tabular}{c|cccc}
\hline \hline Nr. of Iter. & $\Delta=0.2$ & $\Delta=0.5$ & $\Delta=1.0$ & $\Delta=2.0$ \\
\hline 5 & $14: 16$ & $6: 53$ & $4: 12$ & $2: 39$ \\
3 & $11: 15$ & $5: 21$ & $3: 13$ & $2: 02$ \\
\hline
\end{tabular}

\section{Conclusion}

The new results on fully implicit discretizations of the homogeneous Boltzmann equation with Maxwellian molecules leads by simple modifications to a second order accurate scheme in time. For a simple testcase one is able to improve the efficiency of particle schemes based on an explicit discretization with respect to accuracy and computational effort.

One may expect similar results for spatially inhomogeneous problems; it remains to perform numerical experiments in this direction.

The generalization of the fully implicit as well as the second order scheme to the general case - the Boltzmann equation with arbitrary differential cross section - is under investigation. 


\section{References}

H. Babovsky (1989), A convergence proof for Nanbu's Boltzmann simulation scheme, European Journal of Mechanics, B/fluids, 8, No. 1, 41-55.

G.A. Bird (1994), Molecular Gas Dynamics and the Direct Simulation of Gas Flows, Clarendon Press, Oxford.

A.V. Bobylev and J. Struckmeier (1994), Implicit and Iterative Methods for the Boltzmann Equation, Report No. 123, Lab. Technomathematics, University of Kaiserslautern, submitted to Transport Theory and Statistical Physics.

C. Cercignani, R. Illner and M. Pulvirenti (1994), The Mathematical Theory of Dilute Gases, Springer, New York.

H. Neunzert, F. Gropengießer and J. Struckmeier (1991), Computational Methods for the Boltzmann Equation, Venice 1989: The State of Art in Appl. and Ind. Math., ed. R. Spigler, Kluwer, Dordrecht, 111-140.

H. Neunzert and J. Struckmeier (1994), Boltzmann Simulation by Particle Methods, Report No. 112, Lab. Technomathematics, University of Kaiserslautern.

G. Russo and R.E. Caflisch (1994), Implicit Methods for Kinetic Equations, Rarefied Gas Dynamics: Theory and Simulations, Eds. B.D. Shizgal and D.P. Weaver, Progress in Astronautics and Aeronautics, Volume 159.

J. Struckmeier (1995), Implicit Particle Methods for the Boltzmann Equation with Maxwellian Molecules, preprint, Department of Mathematics, University of Kaiserslautern.

Jens Struckmeier and Konrad Steiner

Department of Mathematics

University of Kaiserslautern

P.O. Box 3049

67653 Kaiserslautern

Germany

E-mail: struckm@mathematik.uni-kl.de 